\title{
Mapas conceptuales colaborativos: un modelo basado en grafos distribuidos
}

\author{
Jorge A. Hernández-Perales, Manuel Hernández-Gutiérrez \\ Universidad Tecnológica de la Mixteca, Huajuapan de León, Oaxaca \\ jahdezp@mixteco.utm.mx, manuelhg@mixteco.utm.mx
}

Resumen. Conforme el tiempo transcurre, el acervo de conocimiento humano se hace más y más grande, y con el advenimiento de medios alternativos de comunicación, este conocimiento tiende a fragmentarse y desorganizarse. Los mapas conceptuales basados en grafos permiten que una persona exprese de una forma efectiva, ágil y amena sus conceptos. La tecnología actual también permite que estos mapas puedan compartirse colaborativamente, así logrando crear mapas grupales en los que intervienen los conceptos de varias personas. En este trabajo presentamos un modelo epistemológico para la creación y el manejo de mapas conceptuales distribuidos. Este modelo tiene el propósito de enmarcar en una teoría de grafos con anotaciones temporales la forma en que los humanos adquieren, organizan y clasifican cierto tipo de conocimiento.

Palabras clave: aprendizaje a distancia, grafos, conocimiento, colaboración.

\section{Collaborative Conceptual Maps Based on Distributed Graphs}

\begin{abstract}
As time goes by, human knowledge is getting bigger and bigger, and with the advent of alternative means of communication, this knowledge tends to fragment and get disorganized. Conceptual maps based on graphs allow a person expresses in an effective, agile and entertaining way his/her concepts. Current technology also allows these maps to be shared collaboratively, thus achieving the creation of collaborative maps in which the concepts of several people intervene. In this paper we present an epistemological model for the creation and management of conceptual and distributed maps. This model has the purpose of framing in a theory of graphs with temporal annotations the way in which humans acquire, organize and classify a certain type of knowledge.
\end{abstract}

Keywords: learning, graphs, knowledge representation, collaborative.

\section{Introducción}

La adquisición y la organización del conocimiento humano es una de las actividades principales en el aprendizaje y la enseñanza; también es clave en la 
información compartida y forma una de las bases principales del procesamiento de la información en la inteligencia artificial 9. Tal manejo del conocimiento humano es útil en aplicaciones modernas tales como la web semántica [1, así como en clasificaciones generadas por usuarios que escriben anotaciones (por ejemplo, a imágenes) para después formar redes semánticas. Los mapas conceptuales son objetos gráficos que muestran componentes básicos de información relacionados entre sí. Tales componentes de información entrelazan y condensan conceptos. Una de las formulaciones gráficas de mapas conceptuales es por medio de visualizaciones inspiradas en la teoría de grafos [5], en donde cada nodo de un grafo representa un concepto (expresado mediante una palabra), y en donde cada lado enlaza un nodo con otro si tal par de nodos satisfacen una relación binaria. En este escrito mostraremos que la creación distribuida de mapas conceptuales tiene implicaciones positivas para el manejo estructurado de información, dentro de un modelo epistemológico básico; además, se analizarán y propondrán algunas herramientas para la creación computacional y la construcción distribuida de este tipo de mapas.

Este trabajo está organizado como sigue. En la sección 2 se plantea el concepto de epistemología y el de ontología, y una formulación de esta última como mapa conceptual. En la sección 3 plantearemos una relación que existe entre un mapa conceptual y un grafo. En la sección 4 se plantearán un conjunto posible de estrategias para la construcción de mapas conceptuales, intentando resolver el problema de condición de carrera (race condition) en la creación de grafos colaborativos. En la sección 5 se da una implementación de mapas conceptuales como grafos en Prolog, y en la sección 6. Conclusiones, se describen alcances y limitantes de la modelación; también se finaliza con algunas notas de resumen, se plantean algunas aplicaciones en la educación, y se dan notas de trabajo a futuro.

\section{Ontologías y mapas conceptuales}

Consideraremos en este escrito un cierto concepto de epistemología (definida tradicionalmente como un parte de la filosofía que estudia los principios, extensiones y métodos del conocimiento humano), en una vertiente pragmática parecida a como se menciona "ontología" en los medios computacionales.

Así pues, para nuestros propósitos, definimos un modelo epistemológico relacional como un conjunto $I_{t}$ de información tomada de un universo $U$ con otro conjunto de relaciones $R_{t}$ (de un universo de relaciones $R$ ) definidas binariamente en $I_{t}$, y tal que $t$ está dado como un parámetro de tiempo. La forma en que $I_{t}$ cambia es mediante las operaciones: al eliminar de $U$ uno de sus elementos (eli) o al agregar (ag) a $U$ algún otro elemento, en tanto que $R_{t}$ cambia por eliminar una relación (eliR), agregar una nueva (agR) o bien por modificar una relación existente (modR). Este modelo fundamenta adecuadamente un caso particular, a tratar a continuación, de ontología.

La ontología es una definición formal de tipos, propiedades y relaciones entre entidades que real o fundamentalmente existen para un dominio de discurso en 
particular; en este artículo utilizamos este concepto de ontología para el manejo de conocimiento disperso. Comenzaremos investigando el impacto de la creación de mapas conceptuales en la síntesis de ontologías temáticas. Afirmamos por ahora que los mapas conceptuales son una aproximación básica al concepto de ontología: un mapa conceptual representará, en principio, los conceptos básicos que, a su vez, son enmarcados en otro concepto mayor (quizás metaconcepto), permitiendo con esto ya sea a nivel pedagógico o computacional explorar un espacio de búsqueda conceptual; un mapa conceptual a la vez sirve como una guía tanto a inteligencias artificiales como a la mente humana para decidir entre diversas opciones de exploración dentro del entramado del conocimiento humano; por la parte computacional, un mapa conceptual se puede ver como una forma constructiva de considerar ontologías [8]; por la parte humana, su interpretación da algunas pistas tanto del conocimiento humano como de su posible uso en tareas de razonamiento ([2], [10]). Es necesario enfatizar que los mapas conceptuales distribuidos tienen un alto valor informativo social, pues como se menciona en [6] (p.12-3, 2.1.1.1 Jean Piaget's Pespective of Constructivism), la interacción de cada individuo con su ambiente hace que sus modelos cognitivos sean únicos, y compartirlos hará que los demás participantes incrementen sus propias ideas así como, a su vez, el acervo cognitivo grupal. Nuestra suposición básica, en resumen, es que, dado un modelo epistemológico relacional como el previamente definido para un individuo $A$, las ontologías permiten un manejo y utilización de su acervo epistemológico de tal manera que este acervo se sustenta en consideraciones de conceptos atómicos (palabras) y relaciones binarias entre estos conceptos.

Por razones de eficiencia, los métodos automatizados tales como los de [8] son altamente deseables, aunque si bien su misma propuesta mecanicista indica que la información se auto-contiene y no es posible "incrementar" las posibilidades de mayores conceptos ontológicos, y si fuera posible, la forma desorganizada que adoptarían (por razón del crecimiento exponencial) las haría inútiles para consumo humano. Una mayor estructuración tal como la mencionada en [8], por ser jerárquica, es una buena forma de organizar el conocimiento [10, pero puede considerarse como un caso particular del modelo epistemológico y distribuido propuesto aquí. En suma, cuando la intervención humana es posible, algunos sistemas automatizados mejoran su funcionamiento (como un ejemplo, la adición de etiquetas (tags) por medios sociales a imágenes brinda un contexto para la apropiada clasificación de estas [4]), tal como acontece en redes sociales de orientación gráfica tales como Instagram $^{\complement}$ o Pinterest $^{\complement}$.

\section{Mapas conceptuales como grafos}

En [10] hay una discusión amplia de los mapas conceptuales, sus bases teóricas y algunas de sus aplicaciones; de forma explícita, los grafos trabajados ahí tienen etiquetas en sus lados. Para simplificación de tratamiento, en este artículo omitiremos las etiquetas en los lados.

Modelados mediante grafos, los mapas conceptuales brindan una información considerable en formato bidimensional acerca de un tópico. Aunque tal formato 
es bidimensional por restricciones físicas, es también posible contar con una historia de la creación y desarrollo de un mapa conceptual, así ampliando en una dimensión, la del tiempo, su formulación.

Primeramente, se notará que, por un lado, el número de nodos indicará una densidad informativadel mapa conceptual; por otro lado, el número de lados que unen estos nodos dará una medida de la densidad relacional de este mapa. En un grafo de 5 nodos con todos los nodos conectados entre sí, con el grafo completo llamado $K_{5}$ se tendrá una densidad conceptual moderada, pero una densidad relacional alta, pues por un teorema combinatorio, éste será el mayor número de relaciones establecidas para un grafo completo arbitrario no dirigido $K_{n}: n *(n-1) / 2$.

La elección de un grafo tiene también un acierto computacional: La extracción de la información subyacente es bidireccionalmente sencilla. En efecto, la recolección de información textual y las relaciones es una primitiva en XPCE ${ }^{1}$. por ejemplo, para generar de manera inmediata un conjunto de hechos como programa lógico [1]. De hecho, por medio de diccionarios o listas asociativas (evitando autoreferencias [loops]) de nodos se tendrá listo un archivo de texto para su respectiva versión gráfica.

Diremos que un grafo es un grafo conceptual si tal grafo se enmarca en una historia de mapas conceptuales, cada uno representado por un grafo por el mapeo mencionado previamente. La característica novedosa que es digna de notar en los grafos conceptuales es que estos son objetos gráficos dinámicos, y al agregar la dimensión del tiempo afloran algunos resultados que veremos a continuación. Los grafos conceptuales requieren estar ordenados cronológicamente, y tener por cada grafo convencional anexo una etiqueta de fecha (que puede ser física o de causalidad), así enlazando estos grafos con el modelo epistemológico propuesto. Notemos que un grafo visto como mapa conceptual puede originar varios otros grafos; el grafo conceptual que trabajamos aquí solo toma una posible trayectoria de entre todas las posibles ramificaciones: esta situación resultó inevitable en las consideraciones de modelación basadas en temporalidad.

\subsection{Mapas conceptuales como objetos dinámicos}

Supongamos que cada grafo conceptual se representa con $G_{n}(t)$ para un usuario dado con identidad $n$ en un tiempo $t$ dado. En cada caso, cada $G_{n}(t)$ tendrá asociados sus dos conjuntos principales: Su conjunto de nodos $G_{n}(t)$ [nodos] y su conjunto de lados $G_{n}(t)$ [lados]. La parte pragmática abordada aquí es que los nodos $G_{n}(t)$ representan cierta información (codificada mediante palabras) $I_{t}$, y similarmente, los lados $G_{n}(t)$ [lados] representan algunas relaciones $R_{t}$ que se mantienen entre los nodos de $I_{t}$. Notemos que desde tanto un punto de vista local (por un usuario) como global (por un tablero común, en el caso de un sistema distribuido), el total de nodos se incrementará por cada nuevo usuario que se incorpore, y en posible mayor número, el total de conexiones que se puedan establecer entre estos nodos. De esta manera, algunos de los resultados de teoría

\footnotetext{
${ }^{1}$ Paquete gráfico incluido en Swi-Prolog, www.swi-prolog.org
} 
de grafos solo son aplicables a grafos convencionales que se "congelan" en un tiempo $t_{o}$ (grafos estáticos), pero no a grafos que cambian con el tiempo.

\subsection{Operaciones directas sobre mapas conceptuales}

En esta sección se enfatiza qué operaciones serían posibles sobre grafos que modelan mapas conceptuales, de tal forma que se aporte un estándar de presentación, así como algunas reglas detransformación que incrementen la confianza de un usuario en aportar nuevos conceptos. Describimos algunas operaciones que se pueden obtener mediante la combinación de grafos en el tiempo, teniendo en mente las posibles aplicaciones a mapas conceptuales (ver Fig. 1).

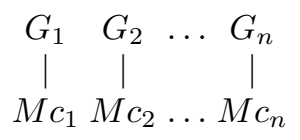

Fig. 1. Grafos (G) relacionados con mapas conceptuales (Mc), donde cada subíndice indica un tiempo $i$ de construcción.

Unión de mapas conceptuales. Ahora, consideremos la unión de dos grafos en la formulación siguiente: La unión de $\operatorname{dos}$ grafos $A$ y $B, A \cup B$ es un nuevo grafo $C$ tal que $C$ tiene como conjunto de nodos el conjunto de nodos de $A$ en unión el conjunto de nodos de $B, C[$ nodos $]=A[$ nodos $] \cup B[$ nodos $]$, y de tal forma que el conjunto de lados de $C$ es igual a la unión del conjunto de lados de $A$ en unión con el conjunto de lados de $B: C[$ lados $]=A[$ lados $] \cup B[$ lados $]$. Esta definición tiene una propiedad epistemológica interesante desde el punto de vista de cada usuario, pues resulta que (bajo el impedimento de borrar nodos o lados ya establecidos) el conocimiento adquirido es siempre monótono (no en un sentido de uniformidad, si no en el sentido de que la información que puede veraz y positivamente concluirse ahora puede concluirse posteriormente, cuando la información se incrementa en el mapa; para casos no monótonos, información adicional puede invalidar conclusiones previas) y consistente. También, conforme $t$ se incrementa y bajo el impedimento del borrado de nodos, es posible considerar que la complejidad del grafo (medida como el número de nodos por el número de lados, que establece una medida adecuada para nuestros propósitos) se incrementa a su vez.

La operación unión y su impacto en los usuarios. Cabe mencionar que al realizarse las operaciones como conjuntos en la unión de grafos, los nodos en común se fusionan automáticamente, así como los lados, y por que además para conjuntos arbitrarios $A \cap B \subseteq A \cup B$. Para etapas posteriores a que se haya diseminado distribuidamente el estado actual de los usuarios (bajo una política de rondas, por ejemplo), cada usuario se concentrará probablemente en una sección del 
grafo total. Esto lleva a un interesante planteamiento de un índice de atención a una sección de un mapa conceptual: Hemos podido comprobar con algunos voluntarios (incluidos los autores) un usuario a lo más ve (con lectura dispersa) 10 nodos simultáneamente, y que toma una decisión conciente de enfocarse en algunos de los 10 elementos para así plantear la creación de nuevos nodos (unos 3 bajo una circunstancia usual de comienzo, ya que generalmente los conceptos también ser irán gastando y terminando, por lo que es necesario considerar que conforme pasa el tiempo habrá mayores retardos para colocar nuevos conceptos), aunque para dar un enfoque medible sería mejor utilizar técnicas de rastreo de la vista (eye tracking) 3], procediendo por medir periodos de vista fija (fixations) y frecuencia de vistazos (saccades), particularmente por que aquí la contigüidad espacial (también mencionada en [3]) entre texto e imágen es difusa, así como el respectivo efecto de desconcentración (split attention). Otros aspectos sicológicos son explorados en [10].

Así que el crecimiento del tiempo $f(n)$ de respuesta (cuando $n$ (instante) crece) está asintóticamente en $\Omega\left(2^{n}\right)$ (haciendo que exista una constante $c, c>0$, con $f(n) \geq c * 2^{n}$ a partir de un número natural dado) y empíricamente nos dice que después de unas cuantas rondas lo mejor que un moderador puede hacer es cerrar los permisos para agregar nuevos nodos, a menos que se abran algunos de estos nodos para continuar agregando elementos (zooming). De todas formas, por la misma base de promover la lluvia de ideas (brainstorming), sesiones cortas de unos minutos parecen la mejor decisión.

Plegado de nodos. Otra operación interesante es la de doblar o plegar (folding) algunos nodos para esperar que posteriormente se abrán a solicitud de un usuario. Nodos que tienes ésta característica pueden tener un indicador de apertura; la idea es que un gran diagrama conceptual probablemente no será requerido en ciertas etapas y puede obviarse su contenido.

Transitividad. Otra operación más es la cerradura transitiva (transitive closure). La operación conlleva la posibilidad de ocultar (no borrar) nodos si tenemos que si $n_{1}, n_{2}$ y $n_{3}$ son nodos, con una nueva relación $R$ sirviendo de conexión entre ellos, con $\left(n_{i}, n_{j}\right) \in R$ si tal conexión es estable, escrita como $n_{i} R n_{j}$, se tiene que $n_{1} R n_{2}$ y $n_{2} R n_{3}$ podríamos implicar $n_{1} R n_{3}$, asumiendo que $R$ es una relación transitiva (un resultado que es alguna veces sorprendente, pero el hecho es que es una conclusión correcta aunque con una pérdida sustancial de información; sería posible, no obstante, agregar un "etiquetado histórico" a cada nodo para evitar tal pérdida de información).

Temporalidad. La última operación que mencionamos es la de incorporar un rastreo temporal, un "estado de edad" de un nodo, donde se asocia secuencialmente una nueva edad a cada nodo (con un contador que se incrementa en cada ocasión). Esta información puede no ser relevante a nivel grupal (a menos que se utilicen relojes lógicos o vectoriales, que son necesarios para propagar en un sistema distribuido la información básica de causalidad [7, Capítulo 3]), pero sí sería necesario que fueran adjuntos a la identidad de un usuario, pues 
la información entonces diseminada sería más profusa y de alguna utilidad (tal como saber qué usuarios participan más activamente, digamos).

\subsection{Trayectorias, subgrafos y árboles generadores}

Explotando un poco más la representación de grafos, es conocido que una trayectoria permite enlazar dos conceptos (nodos) distantes. En un contexto epistemológico la trayectoria es también una fuente importante de estructuración de conocimiento: nos indica una respuesta de cómo dos conceptos se enlazan. En sí, estas trayectorias son también interpretables como subgrafos. Los subgrafos, por otro lado, siendo de índole general, no siempre serán interpretables como conocimiento estructurado. Un ejemplo positivo de subgrafo es la obtención de un concepto y todos sus vecinos adyacentes con algún radio de adyacencia. En el caso de radio 1, estamos caracterizando nodos con forma de estrella: el nodo con sus vecinos inmediatos, que en términos de conocimiento humano parece algo particularmente valioso.

Supongamos, como otro ejemplo, que tenemos un grafo con ciclos como diagrama conceptual. Eso quiere decir que las conexiones son profusas, así que se puede pensar un algoritmo de árbol generador, para de esta manera obtener un nuevo diagrama que mantiene solo las relaciones "esenciales". La pérdida de información subyacente, no obstante, puede no siempre valer la pena en tal posible "optimización", pues la interpretación humana puede valuarse más que meramente la orientación de la información a la máquina. Además, los mismos ciclos pueden detectar casos de razonamiento de petición de principio o de términos primitivos o indefinibles. Los lados de todas formas pueden "ponderarse" (subjetivamente) por parte de los usuarios, en una escala apropiada (sugiriendo 1 a 5, de menor a mayor apreciada "fuerza" de interrelación); bajo este esquema, el árbol generamente maximal dice qué conceptos se asocian "fuertemente" entre sí.

\subsection{Radios y subgrafos radiales}

Como ya se ha mencionado, el concepto de radio en un grafo es también importante en términos de estructuración conceptual. Partiendo del radio 1, los elementos adyacentes están directamente relacionados con el nodo central. Cuando el radio se incrementa, se empieza a perder este tipo de interrelación. Una propuesta básica al respecto es pensar que los mismos radios son una medida objetiva de cercanía conceptual, por lo que empíricamente se puede concluir la siguiente ley de radios al infinito: Cuando el radio $n$ desde un (nodo) punto central $n_{1}$ se incrementa, la relación conceptual entre el nodo $n_{1}$ y aquellos $n_{j}$ ubicados a una distancia $n$ se va perdiendo.

Los subgrafos tienen una interpretación particular en el caso de mapas conceptuales, ya que al no incorporar la información total inicial, pueden originar nuevas formulaciones a partir de su obtención, conduciendo con ello a "ramificaciones" conceptuales (sirviendo como un aliciente de creatividad). Es de notoria relevancia la construcción de subgrafos en su formulación de árboles, dada una visión jerárquica y de lo general-a-particular en la organización de ideas [10. 


\subsection{Desventajas de una representación de grafos}

Algunas desventajas de los mapas conceptuales en la representación mostrada de grafos son heredadas de la simplicidad de los grafos mismos. En los grafos convencionales, la unión mediante un lado de un par de nodos no categoriza en absoluto ni a los nodos ni a los lados: todos los lados tienen la misma jerarquía, e igualmente en los nodos. En el caso de lados ponderados, los valores otorgados pueden ciertamente guiar algún tipo de jerarquía ya sea implícita o explícita, pero aún así los nodos siguen teniendo el problema de carecer de clasificación o de jerarquía.

Otra desventaja detectada es que los grafos rápidamente crecen en un tratamiento individualizado y todavía más si son creados por varios usuarios (en orden exponencial). El significado de esto es que en pocas rondas los usuarios son fácilmente abrumados por la acumulación de información, detractando así la idea de que los mapas conceptuales deberían ser utilizados para organizar información y hacerla humanamente asimilable.

\section{Estrategias de formación de mapas conceptuales colaborativos}

Tres son las estrategias colaborativas para diseñar mc's, en donde cada estrategia propuesta es: la estrategia casual,la estrategia de concurrencia planificada, y la estrategia asíncrona.

Estrategia casual. La estrategia casual procede con la confección independiente de un grafo para posterior fusión, así generando un nuevo grafo que se distribuye para nuevo análisis y diseño. No se permite el borrado de conceptos en cada iteración, siendo preferible comenzar otra sesión si alguno de los colaboradores está en franco desacuerdo con el logro parcial alcanzado. El proceso continúa con los usuarios hasta que un usuario simplemente decide quedarse con su parte tal como en una iteración fue obtenida.

Estrategia de concurrencia planificada. La siguiente estrategia es con concurrencia planificada: $\mathrm{Al}$ agregarse un concepto $A$, se distribuye entre los participantes. En caso de que se agregue otro concepto se verifica si el concepto es igual al de alguien más que lo haya planteado, o bien se agrega tal cual. Notemos que no es necesario borrar nodo alguno. Una heurística sencilla señalará qué tan vívido es un concepto: por el grado total ya sea de incidencia o de extroversión (exterioridad) o ambos. No habrá nodos aislados (solo al inicio hay uno), pero los nodos que tengan un grado 1 durante un buen lapso de tiempo serán conceptos que brindaron poca o nula aportación al trabajo grupal, presumiblemente.

La posible síntesis computacional está también basada en el grado de un nodo: Por medio de una simple exploración sobre cada nodo, incluyendo forzosamente el nodo inicial, se tendrá un conjunto de conceptos que abarcarán tanta importancia como el grado que se haya solicitado. Por ejemplo, si los grados asociados a un concepto son como siguen: 
Concepto inicial: auto

\{auto:1, llantas:2, volante:2: espejos:3, interior: 1\}

se seguirá que las personas tienen un fuerte conocimiento consensado de integrar auto con espejos.

Estrategia sincronizada. La tercera estrategia se formula como un juego de varias personas por turnos, en donde cada persona en cada turno debe o agregar un concepto o enlazar ese concepto con algunos de los existentes. Señalado un número de rondas (distribuidas) el juego terminará con un grafo con riqueza conceptual alta (número de nodos) o riqueza relacional alta (número de lados) o bien con conocimiento profuso (suma de ambas riquezas). El problema con este enfoque, como ocurre frecuentemente en aplicaciones sincronizadas, es que el juego puede bloquearse si alguien decide abandonar el juego o existe una desconexión del grupo.

Una forma de aumentar pesos a los lados es por "palomear" una conexión, así que comenzando con peso de lado 1, cada vez que se confirmara la validez de una conexión el peso aumentaría en uno. Esta estrategia de creación de grafos con pesos brinda una considerable información de la "fuerza" de conexión entre conceptos.

El concepto de arbitraje no se requiere como tal en la creación y desarrollo de mapas conceptuales; sin embargo, sería deseable programar un "monitor" o "moderador" que permita anotar algunas informaciones relevantes con respecto a los usuarios, tal como el tiempo dedicado a desarrollar un mapa conceptual o bien sus contribuciones medidas directamente por los nodos agregados; también, el monitor puede decidir cuando dos nodos son iguales vía la discrepancia de una palabra con otra mediante la distancia de edición por ejemplo (o mediante corrección ortográfica directa) así como por posibles sinónimos con un agente monitor guiado semánticamente.

\section{Implementación local en Prolog}

Se ha realizado para experimentación local, y apoyándose en algunas herramientas gráficas provistas por XPCE de Swi-Prolog, un ambiente para el tratamiento epistemológico vía mapas conceptuales mediante grafos (ver Fig. 2 ). Se tienen planeadas extensiones computacionales tanto de índole experimental mediante Erlang o bien práctica, mediante la utilización de una plataforma educativa a distancia llamada Chamild 2

La parte siguiente es un extracto de la codificación realizada hasta ahora en Prolog:

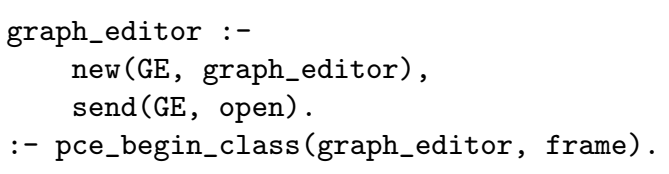

2 https://campus.chamilo.org/ 


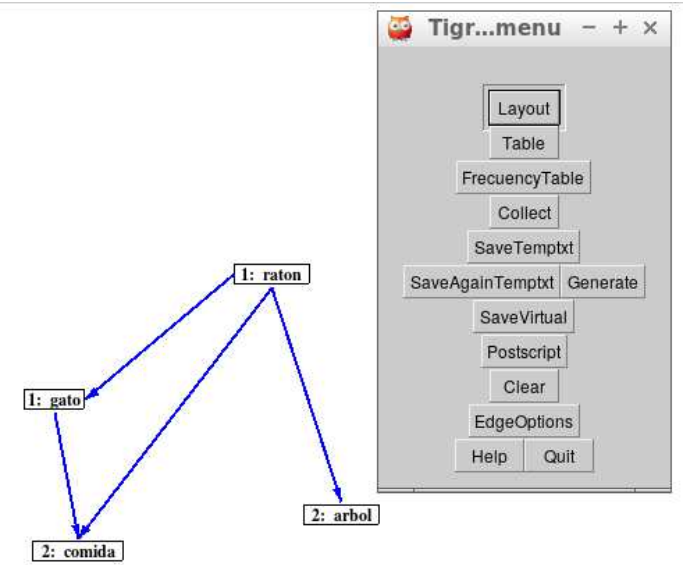

Fig. 2. Una implementación con anotaciones en nodos de un ambiente gráfico para grafos como mapas conceptuales.

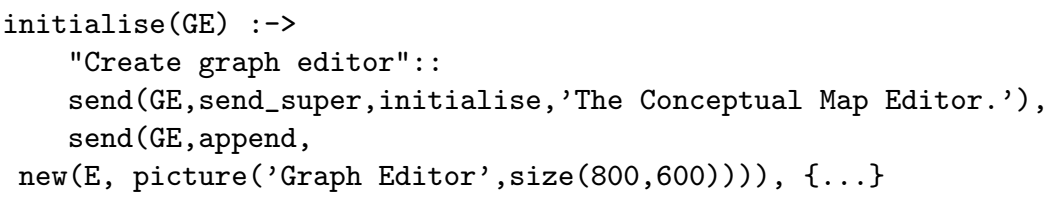

\section{Conclusiones}

Como limitantes detectadas del esquema propuesto se tiene: No hay manejo de información negativa, pues poner información negativa rápidamente saturaría los grafos con lados. Solo información positiva es posible, del tipo "A se relaciona con B", advirtiendo a los usuarios que afirmaciones del tipo "A no se relaciona con B" deberían ser excluídas. Otra limitante es aquella de permitir solo una relación genérica, pero sin duda diversos tipos de relaciones serían epistemológicamente más interesantes y no nos limitaría a solo estructuración simple de ideas. También se hace necesaria de cuando en cuando una "depuración" y "vaciado" de información, ya sea implícita o explícita (para guardar información, el programa propuesto sí puede salvar en una base de datos textual la información introducida); también hemos notado que debería haber algunas técnicas para aminorar la complejidad computacional en el crecimiento de la información y las relaciones.

Algunas aplicaciones posibles a la educación serían: Como moldes para exámenes educativos: Un mapa conceptual parcialmente llenado por completar, por ejemplo; o bien como bases de datos para rescatar conceptos importantes. En el mismo tenor, se puede solicitar la descripción de un concepto y que se de la información y después se relacione con el resto de los componentes. Otra 
aplicación sería la formación de plantillas nodales sin enlaces, para ver qué tanto puede recuperar el usuario. Se puede utilizar la noción de subgrafo para reforzar la comprensión en un tema, solicitando al estudiante la escritura de ensayos mediante los conceptos interrelacionados dados. Se tiene planeado una versión distribuida en dos posibles vertientes: utilizando Erlang para experimentación distribuida, o utilizando el sistema Chamilo (plataforma educativa distribuida de fuente libre) para incrustarle alguna componente que incorpore mapas conceptuales como grafos.

Agradecimientos. Los autores desean expresar un agradecimiento especial a lasautoridades administrativas y académicas de la Universidad Tecnológica de la Mixteca, por su siempre atenta y pronta respuesta a ayudarnos a realizar nuestra investigación.

\section{Referencias}

1. Davies, J., Fensel, D., van Harmelen, F. (eds.): Towards the semantic web: Ontology-driven knowledge managemente. John Wiley \& Sons (2003)

2. Glasgow, J., Narayanan, N.H., Chandrasekaran, B. (eds.): Diagrammatic Reasoning: Cognitive and Computational Perspectives. MIT Press, Cambridge, MA, USA (1995)

3. van Gog, T., Jarodzka, H.: Eye tracking as a tool to study and enhance cognitive and metacognitive processes in computer-based learning environments. In: International Handbook of Metacognition and Learning Technologies. Springer (2013)

4. Johnson, J., Ballan, L., Li, F.: Love thy neighbors: Image annotation by exploiting image metadata. CoRR abs/1508.07647 (2015), http://arxiv.org/abs/1508.07647

5. Jungnickel, D.: Graphs, networks and algorithms. Springer (2008)

6. Konert, J.: Interactive Multimedia Learning. Springer Theses, Springer (2015)

7. Kshemkalyani, A.D., Singhal, M.: Distributed Computing Principles, Algorithms, and Systems. Cambridge University Press (2008)

8. Makagonov, P., Figueroa, A.R., Gelbukh, A.: Natural Language Processing and Information Systems: 11th International Conference on Applications of Natural Language to Information Systems, NLDB 2006, Klagenfurt, Austria, May 31 June 2, 2006. Proceedings, chap. Studying Evolution of a Branch of Knowledge by Constructing and Analyzing Its Ontology, pp. 37-45. Springer Berlin Heidelberg, Berlin, Heidelberg (2006), http://dx.doi.org/10.1007/11765448_4

9. Makagonov, P., Figueroa, A.R., Sboychakov, K., Gelbukh, A.: Learning a domain ontology from hierarchically structured texts. In: Proc. of Workshop Learning and Extending Lexical Ontologies by using Machine Learning Methods. 22nd International Conference on Machine Learning, ICML 2005, August 7-11, 2005, Bonn, Germany (2005)

10. Novak, J., Cañas, A.: La teoría subyacente a los mapas conceptuales y a cómo construirlos (06 2015)

11. Sterling, L., Shapiro, E.: The art of Prolog. The MIT Press (1992) 